\title{
Competitive adsorption of phenol and 3-chlorophenol on purified MWCNTs
}

\author{
Ajna Tóth ${ }^{1}$, Andrea Törőcsik $^{1}$, Etelka Tombácz $^{2}$, Krisztina László $^{1}$ \\ ${ }^{1}$ Department of Physical Chemistry and Materials Science, Budapest University of Technology and Economics, \\ H-1521 Budapest, Hungary \\ ${ }^{2}$ Department of Physical Chemistry and Material Science, University of Szeged, \\ H-6720 Szeged, Aradi Vértanúk tere 1., Hungary
}

\begin{abstract}
A commercial multiwall carbon nanotube and its carboxylated derivate (CNTC and COOHC, respectively) was used after purification to study the competitive adsorption of phenol (P) and m-chlorophenol (CP) from $0.1 \mathrm{M}$ aqueous $\mathrm{NaCl}$ solutions without external $\mathrm{pH}$ control. The adsorption takes place practically exclusively on the external surface of the nanotubes. The uptake of $\mathrm{P}$ is suppressed in comparison to its single solute behaviour on both nanotubes, independently of the initial pollutant concentration. The uptake of $\mathrm{CP}$ however is more sensitive to the concentration and the surface chemistry of the nanotube. The measured coadsorption isotherms were compared to the isotherms calculated from the competitive Langmuir model (CLM). Preferential adsorption of CP was observed in about $95 \%$ of the relative concentration range. The total adsorption may exceed the corresponding single component sorption capacity.
\end{abstract}

\section{Introduction}

For multiple reasons, the sorption behaviour of carbon nanotubes (CNTs) has been intensively studied in the past decade. The expanding carbon nanotube industry is actively seeking new applications. Their large surface area and surface hydrophobicity make CNTs an excellent potential adsorbent of organic pollutants from aqueous media. From an ecological point of view, however, not only engineered CNTs, but also nanotubes are released into the environment through the combustion of fossil fuels. There, they become exposed to natural water systems and can exchange with organic pollutants such as aromatic or polyaromatic hydrocarbons (PAHs) [1-4]. Their high surface area coupled with their mobility can easily modify the transport of various pollutants in any environmental sector. The surface oxygen groups and the $\mathrm{pH}$ may severely enhance the uptake of naphtols due to transformation processes [1-2, 4-5]. No such effect, however, was reported in the case of various phenols, including chlorophenol [2, 4, 6-8]. In spite of a concentrated research effort in the field, the behaviour of CNTs in the environment is not fully known. 
Most sorption studies are performed with aqueous solutions containing only one pollutant. From a practical point of view however, competitive adsorption from binary or multisolute systems is more relevant. Reference works apply one of the following techniques for such investigations: i) the concentration of one of the components is systematically changed while that of the others(s) is kept constant [9-12]; ii) the initial concentration of the pollutants is varied but their molar ratio is kept constant and [9, 13-14]; iii) the total initial concentration of the pollutants is constant but their molar ratios are varied [15-16]. The advantage of the last method is that it makes possible the comparison of the behaviour of solutes in a wide relative concentration range.

A comparative study on the adsorption of aqueous bi-solute monochlorinated phenols on porous carbons led to the conclusion that the surface - aromatic molecule interactions are influenced by the presence of the second solute. While the presence of o- or p-chlorinated phenol suppressed the adsorption of phenol, increasing the concentration of phenol only slightly affected the adsorption capacity for the chlorophenols [11, 14].

On high surface area graphite and activated carbon (AC), phenol and aniline only slightly influenced each other's adsorption. When oxygen functionalities were present the basic compound adsorbs preferentially and this is more pronounced at low adsorbate concentrations [13].

It is widely accepted that results obtained on ACs cannot be extended directly to CNTs. Therefore, the potential applications of the CNTs on an industrial scale, as well as their fate in the environment, initiated an intensive research into the interaction of aqueous solutions of organic impurities with CNTs [1, 3-4, 17-19]. Only a few studies, however, investigate adsorption from multisolute model solutions.

Competitive adsorption of phenanthrene, pyrene and naphthalene were investigated on multiwalled CNTs, non-modified after acidic $\left(\mathrm{HNO}_{3}\right.$ and $\left.\mathrm{H}_{2} \mathrm{SO}_{4}\right)$ purification. All the single-, bi-, tri-solute isotherms could be successfully fitted to the Dubinin -Ashtakhov model. The adsorption of a given primary solute changed from significantly nonlinear to nearly linear when competitors were added. The competitive sorption was found to depend on the relative equilibrium concentrations of the primary and the co-solutes. Significant competition was observed at relatively low concentrations of the primary solute and high concentrations of competitors. Competition was much weaker at relatively high concentrations of the primary solute if the competitors were present in low concentration. The influence of the other solutes was hardly detected when the concentration of the primary solute was close to its solubility limit. As neither pore-filling nor partition-adsorption mechanism explained the behaviour of 
these PAHs, a Polanyi-based surface adsorption mechanism was proposed to interpret the observed sorption and competition [10]. It was also found that the adsorption of 2,4dichlorophenol and 4-chloroaniline either in neutral or ionic form can be supressed by nonpolar naphthalene on multiwalled CNT [8].

In this study we compare the adsorption properties of pristine and $\mathrm{COOH}$ functionalised commercial multiwall nanotubes (MWCNTs) from a two-solute aqueous solution of phenol and 3-chlorophenol. Phenol is the most frequent aromatic contaminant of water and could be easily converted to (poly)chlorinated phenol derivatives in the chlorination step of water purification. Competitive adsorption on O-bearing nanotubes, although they exhibit enhanced wettability with water, was not reported.

\section{Experimental}

\subsection{Carbon nanotubes}

Multi-walled carbon nanotubes, one in its pristine and the other in its carboxyl functionalized form, were purchased from Chengdu Organic Chemicals Co. Ltd., Chinese Academy of Sciences (purity $>95(\mathrm{~m} / \mathrm{m}) \%$ ). Their nominal external and internal diameters are 10-20 and 5-10 nm, respectively, and their length is 10-30 $\mu \mathrm{m}$. According to the manufacturer, the functionalized nanotube contains $2 \mathrm{~m} / \mathrm{m} \% \mathrm{COOH}$. The MWCNTs were purified with aqueous $\mathrm{HCl}$ solution to remove accessible traces of the catalyst [20]. Fulvic acid impurities were removed by the treatment described by Wang et al [21]. The purified samples are labelled as CNTC and COOHC, respectively.

\subsection{Characterisation methods}

\subsubsection{Morphology}

Nitrogen adsorption/desorption isotherms were measured at $77 \mathrm{~K}$, using a Nova 2000e (Quantachrome, USA) computer controlled apparatus. The samples were evacuated at $293 \mathrm{~K}$ for 24 hours. The apparent surface area $S_{B E T}$ was obtained from the BET model. The total pore volume $V_{T O T}$ was calculated from the amount of nitrogen vapour adsorbed at a relative pressure close to 1 on the assumption that the pores are then filled with liquid nitrogen. Micropore volume $\left(W_{0}\right)$ was deduced from the Dubinin-Raduskevich model.

\subsubsection{Acid-base properties in aqueous solution}

The potentiometric titration of the surface functional groups was performed on a laboratorydeveloped computer-controlled titration system [16]. Details of the titration in the case of 
CNTs are described elsewhere [22]. The initial (immersion) $\mathrm{pH}$ of the potentiometric titration is used to characterize the behaviour of the carbon nanotubes in aqueous solution.

\subsection{Adsorption isotherms from dilute aqueous solutions}

Phenol (P, Merck, $99.5 \%$ ) and 3-chlorophenol (CP, Merck, >98 \%) were used as model pollutants. Their relevant physico-chemical properties are listed in Table 1 . They were used in $0.1 \mathrm{M}$ aqueous $\mathrm{NaCl}$ solution. The initial total aromatic concentrations $\left(c_{\text {total, }}, 0\right)$ of the solutions were fixed as $0.417 \mathrm{mM}$ and $1.25 \mathrm{mM}$. The molar ratios of phenol and 3chlorophenol were systematically changed between 0 and 1 . The relative concentration of the solutes $x_{i}$ is defined as

$$
x_{i}=\frac{c_{i}}{\sum_{i} c_{i}}
$$

where $c_{i}$ is the molar concentration of component $i$.

Table 1. Selected physico-chemical data of the aromatic compounds

\begin{tabular}{|l|l|l|}
\hline & Phenol & \\
\hline & 94.11 & 139.16 \\
\hline Molar mass & $82[23]$ & $26[23]$ \\
\hline Solubility in water $(\mathrm{g} / \mathrm{L}), 20^{\circ} \mathrm{C}$ & 8.85 \\
\hline $\mathrm{p} K_{a}, 20^{\circ} \mathrm{C}$ & 9.89 & $0.60 \mathrm{~nm}^{2}[24]$ \\
\hline $\begin{array}{l}\text { Cross sectional area } \\
\left(\mathrm{nm}{ }^{2} / \mathrm{molecule}\right)\end{array}$ & $0.42 \mathrm{~nm}^{2}[16]$ & \\
\hline
\end{tabular}

$10 \mathrm{mg}$ of CNT was sonicated for 15 minutes with $3 \mathrm{ml} 0.1 \mathrm{M} \mathrm{NaCl}$ in tightly closed centrifuge tubes. After $24 \mathrm{hrs} 3 \mathrm{ml}$ pollutant mixture was added, and the tubes were shaken for 3 days in darkness at $293 \mathrm{~K}$ to reach equilibrium. Contact times needed to reach equilibrium were deduced from previous kinetic measurements. After centrifugation the equilibrium concentration was determined by Ultra Performance Liquid Chromatography (UPLC) (Waters). $20 \mu \mathrm{l}$ of sample were analysed on a BEH C18 column (1.7 mm, 50x2.1 
$\mathrm{mm}$ ) using 40:60 methanol:water containing $0.2 \%$ phosphoric acid as eluent at $1.15 \mathrm{ml} / \mathrm{min}$ flow rate. The UV absorption was detected at $272.8 \mathrm{~nm}$. UPLC analysis of P and CP solutions showed no sign of degradation of the aromatic compounds after 3 days.

\section{Results and discussion}

\subsection{Characterisation of the materials}

Low temperature $\mathrm{N}_{2}$ adsorption isotherms (not shown here) are of type IV in the IUPAC classification, with a type H3 hysteresis loop typical of aggregates [25]. The total pore volume, micropore volume and surface area are listed in Table 2. The ratio of the D and G bands $I_{G} / I_{D}$ determined from Raman spectroscopy showed no difference between the two nanotubes: $I_{G} / I_{D}=1.3$ for both purified MWCNTs. $\mathrm{N}_{2}$-adsorption and Raman measurements reveal no significant difference in the morphology of these tubes.

Table 2 Data derived from low temperature $\mathrm{N}_{2}$ adsorption and $\mathrm{pH}$ of the CNTs

\begin{tabular}{|c|c|c|c|c|}
\hline & $\begin{array}{c}S_{\mathrm{BET}} \\
\mathrm{m}^{2} / \mathrm{g}\end{array}$ & $\begin{array}{c}V_{\text {tot }} \\
\mathrm{cm}^{3} / \mathrm{g}\end{array}$ & $\begin{array}{c}W_{0} \\
\mathrm{~cm}^{3} / \mathrm{g}\end{array}$ & $\mathrm{pH}$ \\
\hline $\mathrm{CNTC}$ & 167 & 1.08 & 0.074 & 4.9 \\
\hline $\mathrm{COOHC}$ & 187 & 1.08 & 0.067 & 4.1 \\
\hline
\end{tabular}

The surface chemistry of the CNTs in wet conditions was characterized by the potentiometric titration curves measured in a $\mathrm{CO}_{2}$-free $0.01 \mathrm{M} \mathrm{NaCl}$ solution (Figure 1, Table 2). The point of zero net proton surface excess occurs at approximately $\mathrm{pH} 4.7$ for CNTC, while the surface charge of COOHC is always negative in the range of the investigation $(\mathrm{pH} \mathrm{3-11).} \mathrm{The}$ divergence between the two tubes becomes striking at higher $\mathrm{pH}$ values. The narrow hysteresis loops indicate that the internal bore of the nanotubes hardly participates in the protonation/deprotonation process as the surface proton exchange is relatively fast. In highly microporous carbons the diffusion control of the proton transport results in a much more pronounced irreversibility [26]. Although the tubes were wide enough to accommodate both the solvent and the solutes, the uptake takes place essentially on the external surface of the CNTs. It was also found that, away from the $\mathrm{pH}$ of the intersection, the surface charge is enhanced by increasing the ionic strength. 


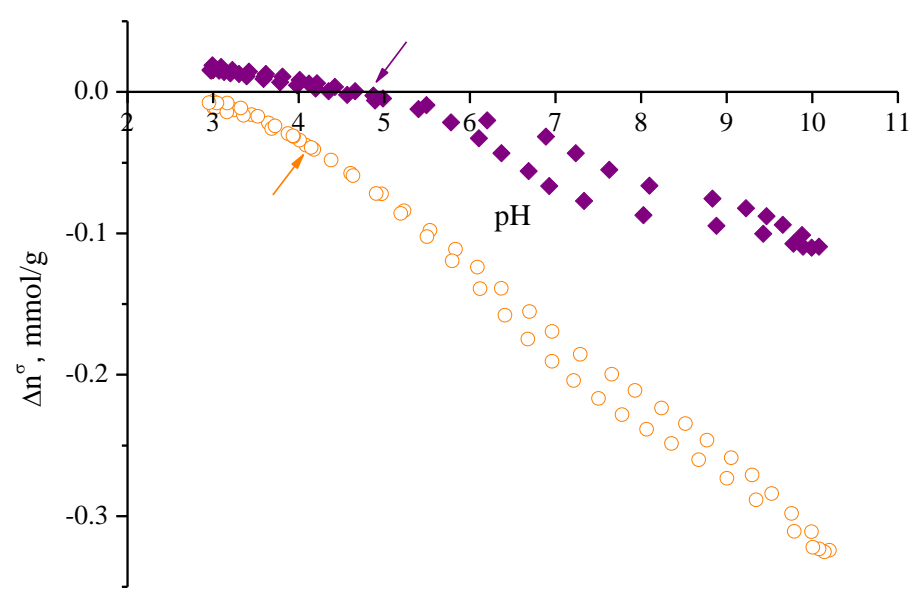

Figure 1. Potentiometric curves of CNTC $(\diamond)$ and $\mathrm{COOHC}(\bigcirc)$. The arrows show the pH of immersion. The specific net proton surface excess amount $n=n_{H^{+}} \quad n_{O H}$ is the difference between the surface excess amounts of $\mathrm{H}^{+}\left(n_{\mathrm{H}^{+}}\right)$and $\mathrm{OH}^{-}\left(n_{\mathrm{OH}}\right)$. Positive values indicate acid consumption, i.e., proton binding from the carbon suspension, while negative values correspond to base consumption, i.e., release of protons or binding of hydroxyl ions. (Color online)

\subsubsection{Single solute isotherms}

The Langmuir model yielded a good fit to the single solute isotherms (not shown here) (Table 3 ). Although increasing the number of the parameters may provide better isotherm fitting [1, $27,28]$ an acceptable fit with meaningful parameters was obtained from the simple Langmuir model.

The adsorption capacity of CP exceeds that of P on both CNTs, which may be explained by the difference in solubility of the two pollutant molecules (Table 1). (Lower solubility means weaker interaction between the solute and the solvent, a principle also used in chromatography.) The initial slopes of the curves are always steeper for $\mathrm{CP}$ as is also reflected in the corresponding $K$ values. Surface area related $n_{m}$ values were used to estimate the surface coverage. It should be noted, however, that the surface area available for the molecules in the liquid phase may be different from $S_{\mathrm{BET}}$ due to i) the kinetic conditions (77 K vs. $293 \mathrm{~K}$ ) and ii) the different cross sectional area of the molecules. The accessibility of the inner borehole during the shaking is to be considered as well [22]. Comparison of the surface area related $n_{m}$ values reveals that the surface coverage by phenol is very similar, $19 \%$ and 
$20 \%$, on the two carbon nanotubes, respectively, i.e., the surface layer also contains a significant amount of the background solution. The adsorption capacities of CP on CNTC and COOHC are also very similar: the surface coverage of CNTC and COOHC by CP is $82 \%$ and $72 \%$, respectively: this nanotube is significantly more densely populated than the nonoxidized one.

Table 3 Langmuir parameters of the single-solute isotherms*

\begin{tabular}{|c|c|c|c|c|c|c|c|c|c|c|}
\hline & \multicolumn{5}{|c|}{ CNTC } & \multicolumn{5}{|c|}{$\mathrm{COOHC}$} \\
\hline & \multicolumn{2}{|c|}{$n_{\mathrm{m}}$} & \multirow{3}{*}{$\frac{K}{\frac{\mathrm{L}}{\mathrm{mmol}}}$} & \multirow[t]{3}{*}{$R^{2}$} & \multirow{3}{*}{$\frac{a_{a v}}{\frac{\mathrm{nm}^{2}}{\text { molecule }}}$} & \multicolumn{2}{|c|}{$n_{\mathrm{m}}$} & \multirow{3}{*}{$\frac{K}{\frac{\mathrm{L}}{\mathrm{mmol}}}$} & \multirow[t]{3}{*}{$R^{2}$} & \multirow{3}{*}{$\frac{a_{a v}}{\mathrm{~nm}^{2}}$} \\
\hline & $\mathrm{mmol}$ & $\mu \mathrm{mol}$ & & & & mmol & $\mu \mathrm{mol}$ & & & \\
\hline & $\mathrm{g}$ & $\mathrm{m}^{2}$ & & & & g & $\overline{\mathrm{m}^{2}}$ & & & \\
\hline $\mathrm{P}$ & $\begin{array}{l}0.13 \\
\pm 0.01\end{array}$ & $\begin{array}{l}0.78 \\
\pm 0.06\end{array}$ & $\begin{array}{c}2.7 \\
\pm 0.3\end{array}$ & 0.9804 & 2.14 & $\begin{array}{c}0.15 \\
\pm 0.01\end{array}$ & $\begin{array}{c}0.80 \\
\pm 0.05\end{array}$ & $\begin{array}{c}1.4 \\
\pm 0.2\end{array}$ & 0.8848 & 1.67 \\
\hline $\begin{array}{l}\mathrm{C} \\
\mathrm{P}\end{array}$ & $\begin{array}{l}0.38 \\
\pm 0.02\end{array}$ & $\begin{array}{l}2.28 \\
\pm 0.12\end{array}$ & $\begin{array}{c}3.7 \\
\pm 0.6\end{array}$ & 0.9487 & 0.73 & $\begin{array}{c}0.37 \\
\pm 0.02\end{array}$ & $\begin{array}{c}1.98 \\
\pm 0.10\end{array}$ & $\begin{array}{c}2.0 \\
\pm 0.3\end{array}$ & 0.9554 & 0.84 \\
\hline
\end{tabular}

$* n_{\mathrm{m}}$ and $K$ are the monolayer capacity and the "adsorption coefficient" from the Langmuir model, respectively; $R^{2}$ is the square of the regression; $a_{a v}$ surface area available for a single molecule

The influence of the surface functionalization is more pronounced in the case of the $K$ parameter (Table 3): there is a factor of almost 2 between the corresponding $K$ values of the two nanotubes. $\mathrm{CP}$ interacts more weakly with the COOHC surface, as the electrostatic interaction of the background electrolyte with the negatively charged $\mathrm{COOHC}$ disturbs the interaction with the aromatic compounds. Wisniewski et al. found that increasing the surface oxygen content significantly reduces the adsorption enthalpy of phenol [28]. These authors also propose that the phenol lying directly on the CNT surface is close to the solid state, and by increasing the oxygen content it progressively approaches a state similar to a supercooled liquid.

The Langmuir parameters in Table 3 were used to calculate the individual isotherms in the bi-solute systems.

\subsubsection{Competitive isotherms}

The competitive adsorption isotherms of $\mathrm{P}$ and $\mathrm{CP}$ from their two-solute solutions were measured at two total concentration values that correspond to the initial section and the saturation range of the single solute isotherms. The $n_{a}$ equilibrium uptakes of both pollutants (individual isotherm) were plotted as a function of $x_{\mathrm{i}}$ (defined by Eq. 1). 
Figures 2-3 show the curves obtained for the two nanotubes.

The shape of the experimental isotherms of $\mathrm{P}$ is concave in all four cases, which indicates that the interaction is weak, independently of the surface chemistry of the nanotube. CP always has a convex initial section in both MWCNTs, a sign of a stronger interaction. On

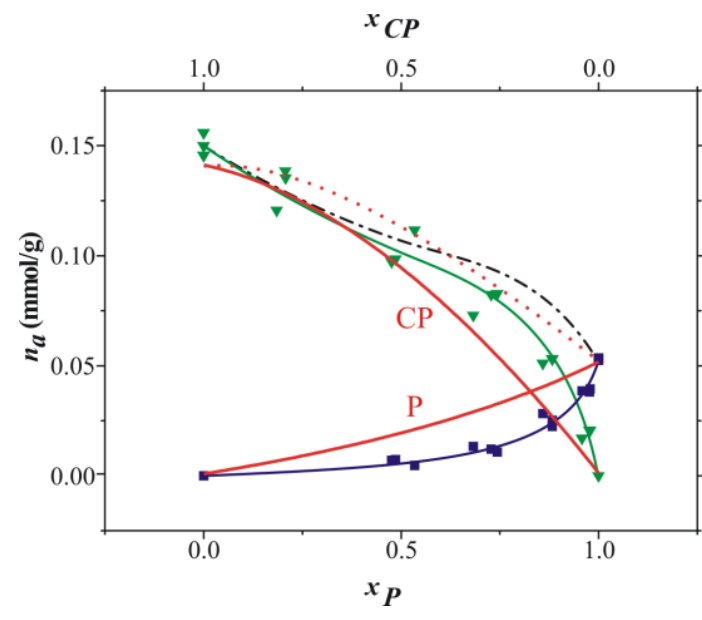

a

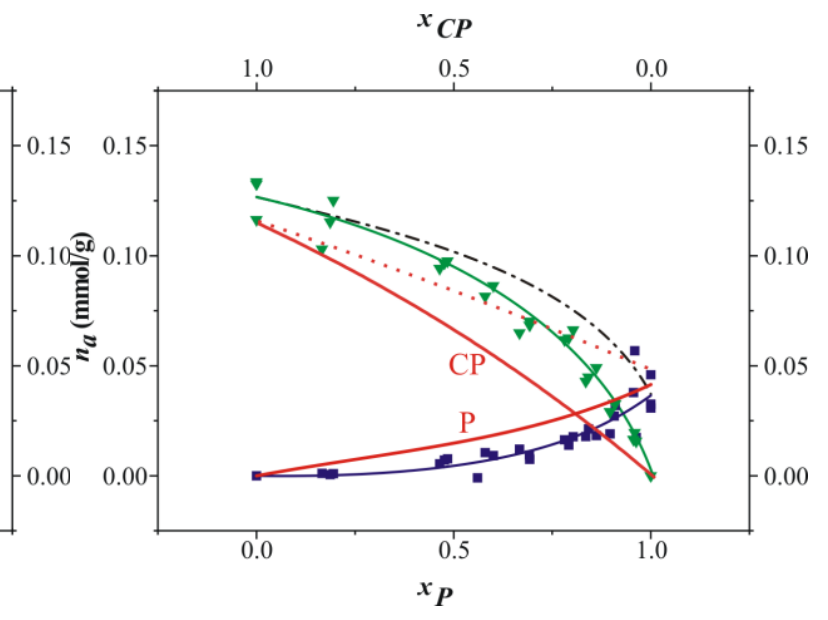

b

Figure 2 Adsorption of phenol ( $\square$ ) and 3-chlorophenol ( $\nabla$ ) from $0.1 \mathrm{M}$ aqueous $\mathrm{NaCl}$ solution on CNTC (a) and COOHC (b). $c_{\text {total }, 0}=0.417 \mathrm{mM}$. The continuous (blue and green) lines are guides for the eye. The (black) dot-dashed line is the algebraic sum of the measured data. The continuous thin (red) lines are the individual isotherms of $\mathrm{P}$ and $\mathrm{CP}$ calculated from Eq. 2. The dotted (red) line is their total. (Colour online)

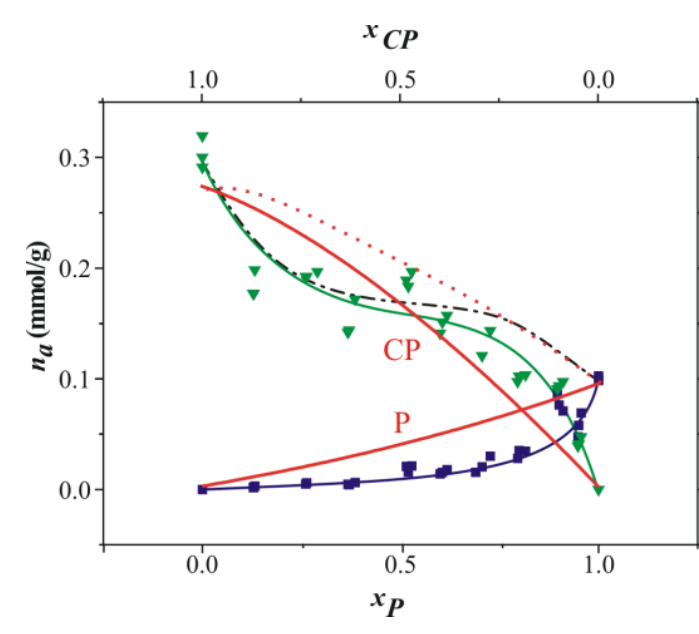

$\mathrm{a}$

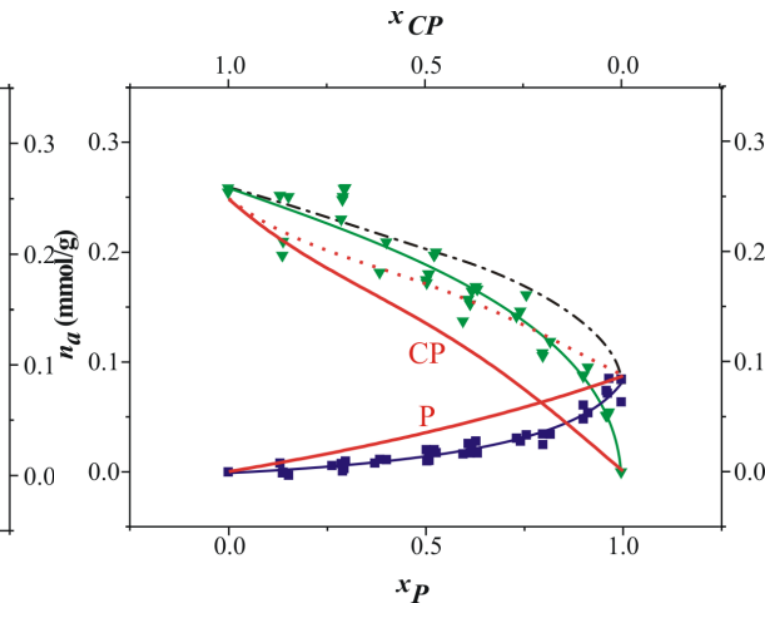

b

Figure 3 Adsorption of phenol ( $\square$ ) and 3-chlorophenol ( $\nabla$ ) from $0.1 \mathrm{M}$ aqueous $\mathrm{NaCl}$ solution on CNTC (a) and COOHC (b). $c_{\text {total }, 0}=1.25 \mathrm{mM}$. The continuous (blue and green) lines are guides for the eye. The (black) dot-dashed line is the algebraic sum of the measured data. The continuous thin (red) lines are the individual isotherms of $\mathrm{P}$ and $\mathrm{CP}$ calculated from

Eq. 2. The dotted (red) line is their total. (Color online) 
CNTC however the shape of the CP isotherm changes with $c_{\text {total }, 0}$. At the higher $c_{\text {total, }, 0}$ the CP isotherm, after a saturation interval, exhibits a very steep increase as $x_{C P} \rightarrow 1$ (Figure 3a). The uptake of the more strongly adsorbing CP has a more pronounced effect on the shape of the total adsorption.

Single solute Langmuir parameters were used to calculate the isotherms according to the competitive Langmuir model (CLM)

$$
n_{a, i}=\frac{n_{m, i} \cdot K_{i} \cdot c_{i}}{1+\sum K_{i} \cdot c_{i}}
$$

The respective calculated curves are shown in Figures 2-3. Comparison of the corresponding measured and calculated curves shows that CLM overestimates the real adsorption of phenol in all the cases studied. Similarly, the real adsorption of CP is underestimated on the oxidized nanotube at both total concentrations (Figure $2 b$ and $3 b$ ). On CNTC, however, the relation of the measured and modelled CP uptake depends on $c_{\text {total }, 0}$. At the lower $c_{\text {total }, 0}$ the measured CP uptake is higher than expected from CLM when $x_{C P}<0.6$, while at higher relative concentrations the real and expected values are practically identical (Figure 2a). At the higher $c_{\text {total, } o}=1.25 \mathrm{mM}$, i.e., on the plateau of the single solute isotherm, at $x_{C P}<0.4$ the real $\mathrm{CP}$ adsorption exceeds the calculated value, but at higher $x_{C P}$ the real uptake is overestimated (Figure 3a). The sigmoidal shape of the individual $\mathrm{CP}$ isotherm at $c_{\text {total }, 0}=1.25 \mathrm{mM}$ indicates that a low concentration of $\mathrm{P}$ already limits the adsorption of CP on this CNT.

As a consequence of these observations the total adsorption on the oxidised CNT is underestimated by the CLM at both total concentrations. In the case of CNTC over- and underestimation may occur equally, depending on the total concentration of the pollutant and their relative concentration. It is worthwhile to mention that Yang et al [10] applied the Polanyi-based Dubinin-Astakhov theory for the competitive adsorption of PAH molecules on a non-functionalized CNT (similar to CNTC). The adsorption calculated from the individual isotherms always overestimated the experimental data. The adsorption of chlorinated phenol and chlorinated aniline on the same CNT was also over-predicted by the Polanyi-based model [8].

The composition of the interfacial layer $x_{\text {layer }, i}$ was calculated as

$$
x_{\text {layer }, i}=\frac{n_{a, i}}{\sum n_{a, i}},
$$

where $n_{a, i}$ is the adsorbed amount of $\mathrm{P}$ or $\mathrm{CP}$, respectively. The relationship between the $x_{i}$ values in the solution and in the interface (Figures 4-5) shows that CP is the preferentially 
adsorbed pollutant on both nanotubes except in a very narrow relative concentration window. The cross-over occurs at $x_{P} \approx 0.95$, i.e., $\mathrm{CP}$ adsorbs preferentially when its relative concentration exceeds $0.05=x_{C P}$, independently of the absolute and relative composition of the free liquid phase and the surface properties of the MWCNT.
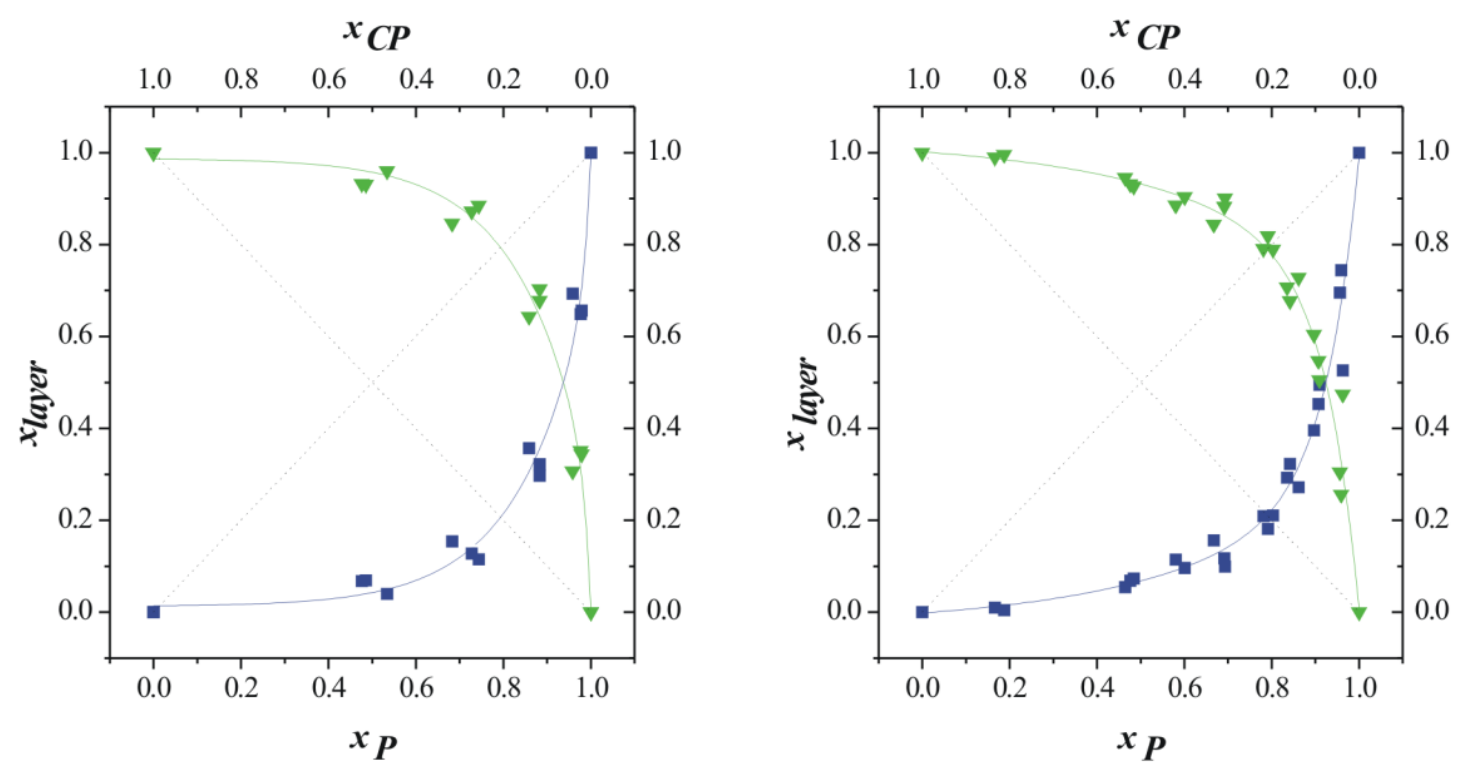

Figure 4 Composition of the interface as a function of relative composition of the bulk liquid phase, $c_{\text {total }, ~}=0.417 \mathrm{mM}$, a) CNTC, b) COOHC; $\square$ phenol, $\nabla 3$-chlorophenol. Solid lines are guides for the eye. (Color online)
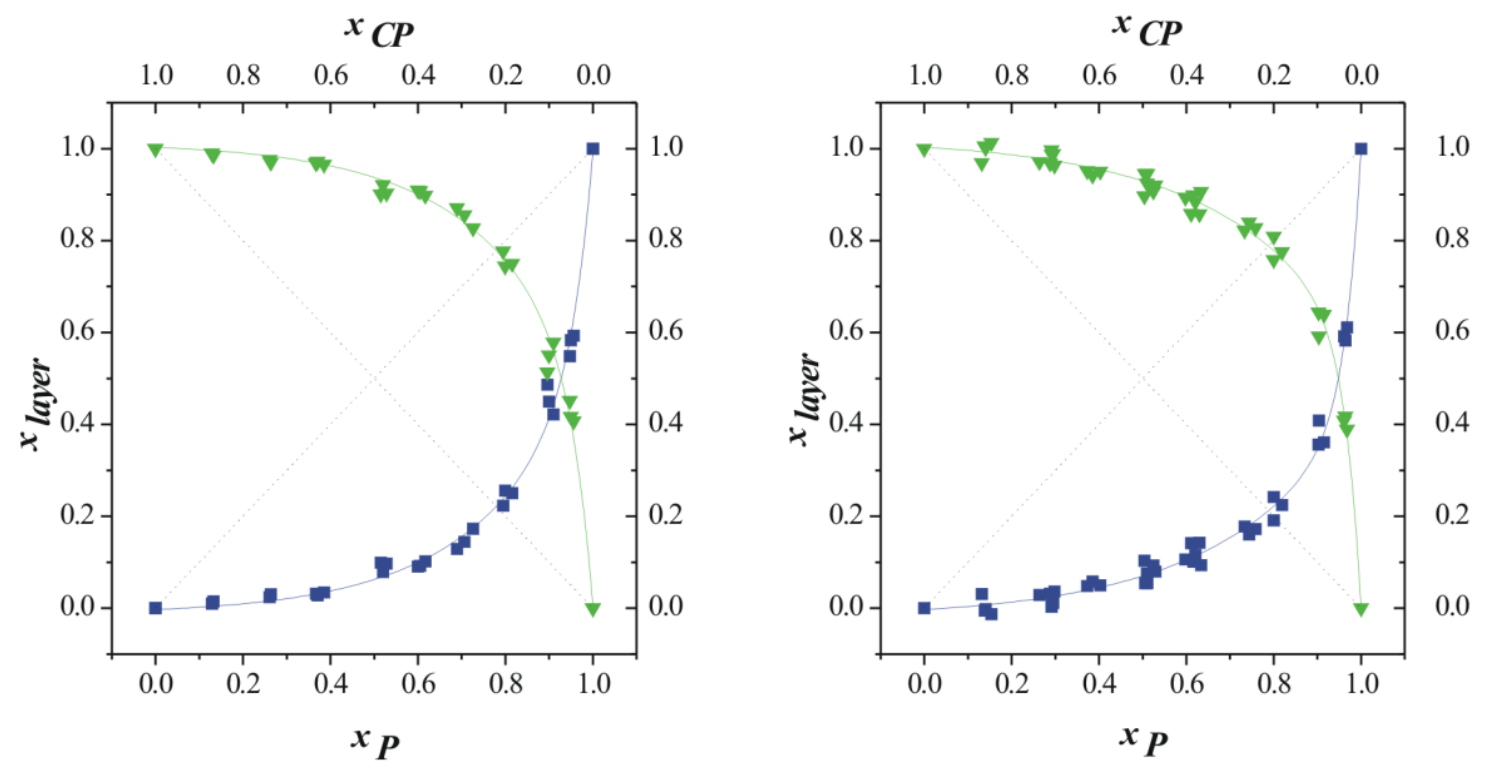

Figure 5 Composition of the interface as a function of relative composition of the bulk liquid phase, $c_{\text {total }, ~}=1.25 \mathrm{mM}$, a) CNTC, b) COOHC; phenol, $\nabla 3$-chlorophenol. Dotted and solid lines are guides for the eye. (Color online) 
For the interpretation of these observations the interactions of the following components must be considered: the surface of the nanotubes, the aromatic probe molecules, the ions from $\mathrm{NaCl}$ and the water. The $\mathrm{pH}$ drops from the initial 5.8-6.4 to 5.3-6.2 and from 5.8-6.5 to 4.0-4.5 in the aqueous phases separated from CNTC and COOHC, respectively. Under these conditions the surface of the CNTC is still close to neutral, while according to Figure 1, COOHC is deprotonated. Both phenols are practically in the protonated, i.e., neutral state. Thus, the most typical interaction between these aromatic species and the CNTs is the $\pi$ $\pi$ interaction between the delocalised electrons $[6,19]$. This interaction is enhanced by the chlorine substituent due to its high electronegativity, as is also indicated by the higher $K$ values of the CP isotherms. Recent molecular model calculations on AC and dilute aqueous phenol solution indicate that the phenol interaction with the surface groups is negligible due to the strong competition with the water [29]. Our experimental data on CNTs also show that the $\mathrm{COOH}$ groups in this concentration do not influence the phenol uptake. The effect of the salt on the adsorption of carbon nanoparticles is a complex matter [17, 30]. As we are very close to the point of zero charge of the CNTC nanotube, the effect of the $\mathrm{NaCl}$ can be neglected here. On the more negative $\mathrm{COOHC}$ surface however, the electrostatic screening of the $\mathrm{Na}^{+}$and $\mathrm{Cl}^{-}$ions may reduce the strength of the $\pi-\pi$ interaction between the CNT surface and the aromatic molecule and reduce the $\mathrm{K}$ value in comparison to the CNTC.

The surface coverage by the aromatic molecules can be characterised by the average surface area available for a single molecule $a_{a v}$ :

$$
a_{a v}=\frac{S_{B E T}}{n_{a} \cdot N_{A}}
$$

where $S_{B E T}$ is the surface area from $\mathrm{N}_{2}$ adsorption, $n_{a}=f\left(x_{P}\right)$ is the total adsorbed amount of phenols and $N_{A}$ is Avogadro's number. Figure 6 shows these values as a function of the relative concentration in the equilibrium free liquid phase.

The population of the surface by the two phenols is a function of the relative composition of the equilibrium liquid phase and the total concentration of the initial solutions. When both aromatic molecules are present at the interface $\left(x_{C P}<0.95\right)$, the adsorbed layer is less ordered, the surface is less populated: the surface area available for a molecule is larger. The 'perturbing' effect of $\mathrm{P}$ is more significant at the lower total concentration. The functionalised nanotube is more sensitive to relative composition, which may be explained by the higher surface charge, and which also may attract the ions of the background salt, thereby disturbing the $\pi-\pi$ interaction and increasing the disorder of the adsorbed layer. 

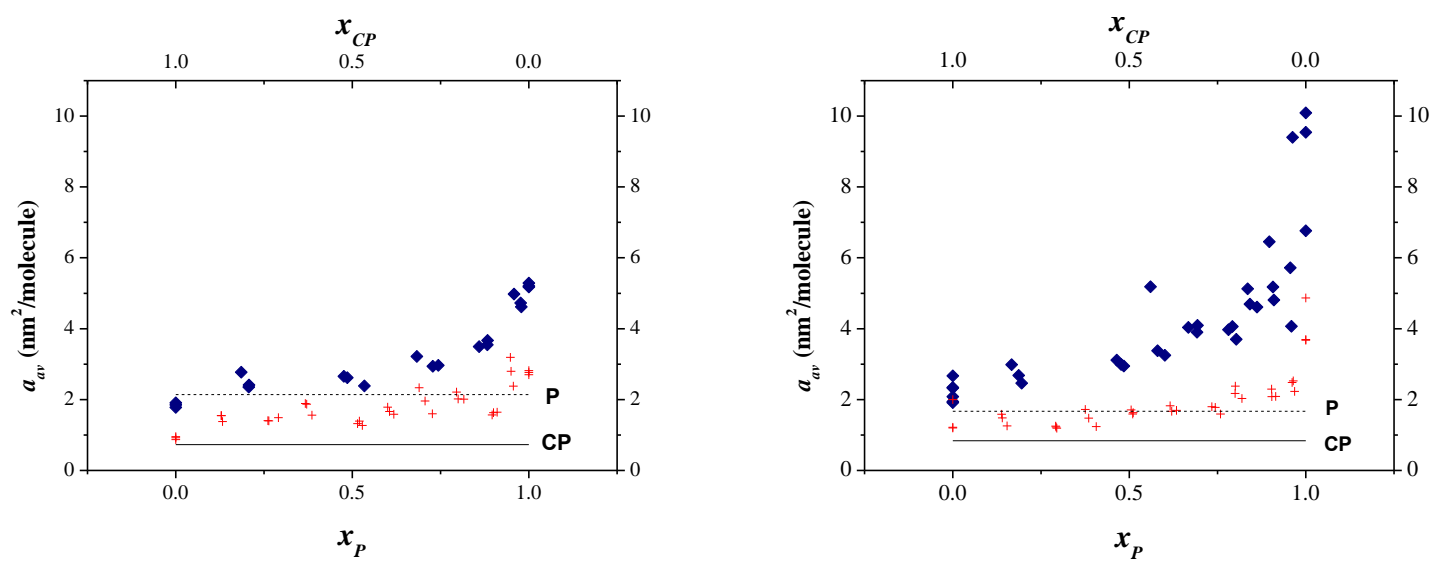

Figure 6 Average surface available $\left(a_{a v}\right)$ for 1 molecule at $c_{\text {total, },}=0.417 \mathrm{mM}(\bullet)$ and $c_{\text {total, }}=$ $1.25 \mathrm{mM}(+)$, a) CNTC, b) COOHC. The two lines indicate the $a_{a v}$ values of $\mathrm{P}$ and $\mathrm{CP}$ from the single-solute isotherms on the two nanotubes. (Color online)

\section{Conclusions}

The results of co-adsorption measurements of phenol $(\mathrm{P})$ and $\mathrm{m}$-chlorophenol $(\mathrm{CP})$ on the two purified commercial CNTs revealed that the following parameters play a significant role in the performance: surface chemistry of the CNT, the chemical nature of the aromatic compound, as well as their total and relative concentration. No external $\mathrm{pH}$ control was applied. Preferential adsorption of the CP was observed in all the systems, a finding that may be explained by its poorer solubility in water. This compound was also more sensitive to the concentration and the surface chemistry of the CNTs. On the oxidized nanotubes the coadsorption resulted in a total uptake at both absolute concentrations that was much higher than expected from the single solute behaviour of the two aromatic molecules. On the plain nanotube however the total adsorption deviated from the expected uptake either in a positive or a negative direction, depending on the relative concentration of $\mathrm{P}$. P may be able to suppress the $\mathrm{CP}$ adsorption already at low concentration. Although the Langmuir model gives a good fit for the single component isotherms, the parameters obtained fail to describe the coadsorption of $\mathrm{P}$ and $\mathrm{CP}$ in the complex systems.

\section{Acknowledgments}

The support of the FP7 Marie Curie IRSES program ENSOR (PIRSES-GA-2010-IRSES269267) and OTKA (K 101861) is gratefully acknowledged. This work is related to the scientific program "Development of quality-oriented and harmonized R+D+I strategy and functional model at BME" supported by the New Széchenyi Development Plan (Project ID: 
TÁMOP-4.2.1/B-09/1/KMR-2010-0002). We express our gratitude to P. Jenei for the UPLC analysis and to Gy. Bosznai for his technical assistance. The scientific discussions with E. Geissler are appreciated.

\section{References}

[1] K. Yang, L. Z. Zhu, B. S. Xing, Environ. Sci. Technol. 40 (2006) 1855.

[2] W. Chen, L. Duan, L.L. Wang, D.Q. Zhu, Environ. Sci. Technol. 42 (2008) 6862.

[3] D. Lin, B. Xing, Environ. Sci. Technol. 42 (2008), 7254.

[4] W. Wu, W. Jiang, W. Xia, K. Yang, B. Xing, J. Coll. Int. Sci. 374 (2012) 226.

[5] K.G. Karhikeyan, J. Chorover, J. M. Bortiansky, P.G. Hatcher, Environ. Sci. 33 (1999) 4009.

[6] K. Yang, W. Wu, Q. Jing, L. Zhu, Env. Sci. Techn. 42 (2008) 7931.

[7] Q. Liao, J. Sun, L. Gao, Carbon 46 (2008) 553

[8] K. Yang, W. Wu, Q. Jing, W. Jiang, B. Xing, Env. Sci. Techn. 44 (2010), 3021.

[9] R. Mihalache, I. Peleanu, I. Meghea, A. Tudorache, J. Radioanal. Nucl. Chem. 229 (1998) 133.

[10] K. Yang, X. Wang, L. Zhu, B. Xing, Env. Sci. Techn. 40 (2006) 5804.

[11] A.R. Khan, T.A. Al-Bahri, A. Al-Haddad, Wat. Res., 31 (1997) 2102.

[12] F. Haghseresht, S. Nouri, G. Q. M. Lu, Carbon 41 (2003) 881.

[13] D. M. Nevskaia, E.Castillejos-Lopez, A. Guerrero-Ruiz, V. Muñoz, Carbon 42 (2004) 653.

[14] Ö. Aktas, F. Çeçen, Adsorption 13 (2007) 159.

[15] J.J. Kipling, Adsorption from Solutions of Non-Electrolytes, Chapter 9, Adsorption in Multicomponent Systems, Academic Press, 1965.

[16] K. László, A. Szücs, Carbon 39 (2001) 1945.

[17] L. Ji, W. S. Chen, Zheng, Z. Xu, D. Zhu, Langmuir 25 (2009) 11608.

[18] B. Pan, B. Xing, Environ. Sci. Technol. 2008, 42 (2008) 9005.

[19] X. Ren, C. Chen, M. Nagatsu, X. Wang, Chem. Eng. J. 170 (2011) 395.

[20] B. Bendjemil, E. Borowiak-Palen, A. Graff, T. Pichler, M. Guerioune, J. Fink, M. Knupfer, Appl. Phys. A 78 (2004) 311.

[21] Z. Wang, M. D. Shirley, S. T. Meikle, R. L. D. Whitby, S. V. Mikhalovsky, Carbon 47 (2009) 73.

[22] A. Tóth, A. Törőcsik, E. Tombácz, E. Oláh, M. Heggen, C. Li, E. Klumpp, E. Geissler, K. László, J. Coll. Int. Sci. 364 (2011) 469.

[23] www.merck-chemicals.com 
[24] C. Borra, A. di Corcia, M. Marchetti, R. Samperi, Anal. Chem. 58 (1986) 2048.

[25] F. Rouquerol, J. Rouquerol, K. Sing, Adsorption by powders and porous solids, Academic Press, 1999, p. 204.

[26] K. László, E. Tombácz, P. Kerepesi, Colloids and Surfaces A 230 (2004) 13.

[27] M. Sander, J. J. Pignatello, Environ. Sci. Technol. 39 (2005) 1606.

[28] M. Wisniewski, A.P. Terzyk, P.A. Gauden, K. Kaneko, Y. Hattori, J. Coll. Int. Sci. 381 (2012) 36 .

[29] I. Efremenko, S. Moshe Langmuir 22 (2006) 3614.

[30] R.L.D. Whitby, V. M.Gun’ko, A. Korobeinyk, R. Busquets,; A.B. Cundy, K. László, J. Skubiszewska-Zięba, R. Leboda, E. Tombácz, I. Y. Tóth, K. Kovács, S. V. Mikhalovsky, ACS Nano 6 (2012) 3967. 